\title{
Identification of novel methylated targets in colorectal cancer by microarray analysis and construction of co-expression network
}

\author{
DONGSHENG LI, JIALIN GUO, SONG WANG, LIANGCHEN ZHU and ZUGANG SHEN
}

Department of General Surgery, Tongji Hospital, Tongji University School of Medicine, Shanghai 200065, P.R. China

Received January 26, 2016; Accepted January 17, 2017

DOI: $10.3892 / \mathrm{ol} .2017 .6506$

\begin{abstract}
The present study was conducted to investigate novel methylated targets in colorectal cancer (CRC). The mRNA expression profiles of GSE32323 in 17 cancer and non-cancerous tissues from CRC patients, as well as expression profiles of 5 CRC cell lines prior and subsequent to 5-aza-2'-deoxycytidine (5-aza-dC) treatment, were obtained from the Gene Expression Omnibus database. The differentially expressed genes (DEGs) in $5 \mathrm{CRC}$ cell lines prior and subsequent to 5 -aza-dC treatment were combined with the CRC-specific gene expression profiling array data. Context likelihood of relatedness algorithm was used to construct the co-expression network of CRC-specific gene expression profile. A sub-network of identified reverse-overlapped DEGs was selected and underwent Kyoto Encyclopedia of Genes and Genomes Pathway Analysis. A total of 6 reverse-overlapped DEGs were identified. This present study verified fibulin 2 (FBLN2) and protein phosphatase 1 regulatory inhibitor subunit 14A (PPP1R14A) to be downregulated in the CRC tissue sample but upregulated in CRC cell lines following 5-aza-dC treatment. The identified reverse-overlapped DEGs were enriched in tumor-associated signaling pathways, including cellular tumor antigen p53, cell cycle and NOD-like receptor (NLR) signaling pathway. A total of 2 silenced genes with abnormal methylation in CRC were identified, including FBLN2 and PPP1R14A. The reverse-overlapped DEGs were enriched in p53, cell cycle and NLR signaling pathways, indicating that reverse-overlapped DEGs, particularly FBLN2 and PPP1R14A, may be important tumor suppressors and that these reverse-overlapped DEGs are inactivated by methylation in CRC.
\end{abstract}

\section{Introduction}

Colorectal cancer (CRC) is one of the most common types of cancer in men and women, with $\sim 1.5$ million new cases and

Correspondence to: Dr Zugang Shen, Department of General Surgery, Tongji Hospital, Tongji University School of Medicine, 389 Xincun Road, Shanghai 200065, P.R. China

E-mail: shenzugang@hotmail.com

Key words: colorectal cancer, 5-aza-2'-deoxycytidine, methylation, co-expression network, context likelihood of relatedness algorithm $\sim 0.5$ million mortalities having been reported in 2013 in the United States (1). In recent decades, the mortality caused by CRC has decreased dramatically owing to the great improvement in early diagnosis and treatment (2). However, CRC remains a prominent global health problem that may be attributed to the lack of comprehensive and systemic understanding of the underlying molecular mechanisms of carcinogenesis.

The accumulation of specific genetic and epigenetic changes is considered to be the main molecular mechanism of tumorigenesis, as it can provide a selective growth advantage of tumor cells over neighboring normal cells (3). Among the epigenetic changes, the abnormal methylation of promoter $\mathrm{CpG}$ islands leading to the transcriptional inactivation of tumor suppressors is considered to be a common mechanism in several human malignancies including CRC (4). Epigenetic masking may participate in the cancerous transformation of colorectal epithelium by affecting the expression of tumor suppressor genes (4). Recent progress in CRC epigenetics studies indicated DNA methylation occurs in the early phase of cancer formation and in the premalignant phase of the adenoma-carcinoma sequence (5). Thus, identifying the epigenetic alterations would be of great value in the early detection of cancers and cancer relapse, as well as in monitoring the response of cancers to therapies (6).

Epigenetically silenced genes by hypermethylation can be reactivated by 5-aza-2'-deoxycytidine (5-aza-dC), which is able to inhibit DNA methylation (7). In addition, the re-expression of silenced genes caused by 5 -aza-dC has been demonstrated in various types of tumors in a dose- and duration-dependent manner (7). The application of 5-aza-dC in expression microarray analysis is considered to be a useful approach for identifying cancer-associated methylated genes (8).

In order to elucidate silenced genes with abnormal methylation in CRC, Khamas et al (9) performed a genome-wide expression screening in $5 \mathrm{CRC}$ cell lines prior and subsequent to 5-aza-dC treatment, and subsequently combined the data with CRC-specific gene expression profiling array. The gene expression data set established by Khamas et al (9) was submitted to the Gene Expression Omnibus (GEO) with the accession number GSE32323. In the present study, the microarray was downloaded and analyzed to identify potential targets for 5-aza-dC by oligonucleotide microarray analysis. A co-expression network of CRC-specific gene expression profile was constructed using the context likelihood of relatedness (CLR) algorithm to identify the signaling pathways in 
which these targets were involved, thus revealing the function of the selected identified genes.

\section{Materials and methods}

Affymetrix microarray data. Transcriptional profile of GSE32323 (9) was extracted from the GEO database (http://www.ncbi.nlm.nih.gov/geo/), which was based on the platform of Affymetrix Human Genome U133 Plus 2.0 Array. A total of 44 chips were available for further analysis, including 17 pairs of cancer and non-cancerous tissues from CRC patients, and expression profiles of $5 \mathrm{CRC}$ cell lines.

Data preprocessing. The raw probe-level data in CEL files were initially converted into expression measures. Robust multiarray average background correction, quantile normalization and probe summarization were subsequently performed in the R (version: 3.0.3, March, 2014) affy package (http://www. bioconductor.org/packages/release/bioc/html/affy.html) (10), and the processed expression matrixes were acquired. For each sample, the expression values of all probes for a given gene were expressed as a single value by taking an average of the values.

Differentially expressed genes (DEGs) analysis. The limma (11) package (http://www.bioconductor.org/packages/2.9/bioc/html/limma.html) in $\mathrm{R}$ was used to identify DEGs in the present study. The following thresholds were set for filtering DEGs: $\log _{2}$ fold-change $(\mathrm{FC}) \mid>1.0$ and $\mathrm{P}$-value $<0.05$. The original P-values were adjusted using Benjamini-Hochberg procedure to correct for multiple comparisons. For CRC cell lines, gene differential expression was calculated from each sample prior and subsequent to 5 -aza-dC treatment. Only DEGs with co-upregulated or co-downregulated expression in $\geq 3$ cell lines were selected and grouped as 'DEGl'. For CRC tissues, DEGs in CRC tissue samples compared to non-cancerous tissue were identified and grouped as 'DEG2'. A comparison was subsequently performed between 'DEG1' and 'DEG2'. The DEGs that simultaneously upregulated in 'DEG1' and downregulated in 'DEG2', or simultaneously downregulated in 'DEG1' and upregulated in 'DEG2' were defined as reverse-overlapped DEGs, and were screened for further analysis.

Co-expression network inference and analysis. To identify interactions between genes, the CLR algorithm was used to construct the co-expression network (DEG2.CEN) in the CRC tissue samples. The CLR threshold was set as 2.5. The sub-network (roDEG.CEN) that associated with reverse-overlapped DEGs was selected from DEG2.CEN by employing the package MINET (http://www.bioconductor. org $/$ packages $/ 3.4 / \mathrm{bioc} / \mathrm{html} / \mathrm{minet}$.html) (12) implemented in R/Bioconductor (version: 3.4; http://www.bioconductor.org/) and subsequently visualized using Cytoscape (version 3.4.0; http://www.cytoscape.org/) (13).

The CLR algorithm (14) is an extension of the relevance network approach, which increases the contrast between physical interactions and indirect associations and takes into account the context of each interaction and association. Links are assigned based on the mutual information (MI) that can accommodate non-linear associations between pair-wise gene expression patterns. The most probable interactions are those whose MI scores stand significantly above the background distribution of MI scores. The MI for two discrete random variables $\mathrm{X}$ and $\mathrm{Y}$ is defined as:

$$
M I=I(X ; Y)=\sum_{i, j} P\left(x_{i}, y_{j}\right) \log \frac{P\left(x_{i}, y_{j}\right)}{P\left(x_{i}\right) P\left(y_{j}\right)}
$$

where $\mathrm{x}_{\mathrm{i}}, \mathrm{y}_{\mathrm{j}}$ represent ith and jth expression level of $\mathrm{X}$ and $\mathrm{Y}$, respectively. $\mathrm{P}(\mathrm{xi})$ and $\mathrm{P}(\mathrm{yj})$ are the marginal probability distributions. $\mathrm{P}\left(\mathrm{x}_{\mathrm{i}}, \mathrm{y}_{\mathrm{j}}\right)$ is the joint distribution that the expression levels of $\mathrm{X}$ and $\mathrm{Y}$ are $\mathrm{x}_{\mathrm{i}}$ and $\mathrm{y}_{\mathrm{j}}$, respectively (14).

Pathway enrichment analysis. The Database for Annotation Visualization and Integrated Discovery (15) provides a comprehensive set of functional annotation tools to elucidate biological meaning behind large lists of genes or proteins. Kyoto Encyclopedia of Genes and Genomes (KEGG) analysis was performed in the present study for functional pathway enrichment of reverse-overlapped DEGs with $\mathrm{P}<0.05$ selected as a cut-off criterion. In addition, the enriched functional pathways of reverse-overlapped DEGs and roDEG.CEN were integrated. Thus, a reverse-overlappedDEG would be correlated with a particular enriched functional pathway if the neighboring genes of this particular reverse-overlapped DEG were involved.

\section{Results}

Identification of DEGs. For database GSE32323 $\left(\log _{2} \mathrm{FCl}>1.0\right.$; $\mathrm{P}<0.05$ ), a total of 59 DEG1 $\mathrm{s}$ in five CRC cell lines prior and subsequent to 5 -aza-dC treatment, including 48 upregulated and 11 downregulated genes, were identified. A total of 1,341 DEG2 s with 675 upregulated and 666 downregulated genes were selected when CRC and normal tissue samples were compared. Following comparing between the 'DEG1' and 'DEG2' groups, 10 reverse-overlapped DEGs were selected (Table I). Among the identified reverse-overlapped DEGs, 6 genes [amine oxidase, copper containing 3, fibulin-2 (FBLN2), uridine phosphorylase 1, cysteine-rich protein 1, protein phosphatase 1, regulatory inhibitor subunit $14 \mathrm{~A}$ (PPP1R14A; CPI-17) and heat shock $70 \mathrm{kDa}$ protein 2] were downregulated in CRC tissue sample, but upregulated in CRC cell lines following treatment with 5 -aza-dC.

Co-expression network of DEGs. The co-expression network of DEGs (DEG2.CEN) was constructed by employing the CLR algorithm. The co-expression network was based on the DEG2 expression profile in the CRC tissue samples and the sub-network (roDEG.CEN) that correlated with the identified reverse-overlapped DEGs (Fig. 1). There were 374 nodes and 567 edges in roDEG.CEN. The number of edges emerging from a node was set as the degree of a DEG, as shown in Table I.

Functional pathway analysis of network. Following integrating the roDEG.CEN with the enriched functional pathway, the downregulated genes were enriched in the drug metabolism pathway, while the upregulated genes were enriched in the cellular tumor antigen p53, cell cycle, oocyte meiosis and 
Table I. The characteristics of identified reverse-overlapped differentially expressed genes.

\begin{tabular}{|c|c|c|c|c|}
\hline Gene & EntrezID & Name & ${ }^{\text {aDE_State }}$ & Degree \\
\hline $\mathrm{AOC} 3$ & 8,639 & Amine oxidase, copper containing 3 & down & 76 \\
\hline FBLN2 & 2,199 & Fibulin 2 & down & 66 \\
\hline UPP1 & 7,378 & Uridine phosphorylase 1 & down & 64 \\
\hline MIPEP & 4,285 & Mitochondrial intermediate peptidase & up & 63 \\
\hline RRM2 & 6,241 & Ribonucleotide reductase M2 & up & 59 \\
\hline KIF11 & 3,832 & Kinesin family member 11 & up & 54 \\
\hline PPP1R14A & 94,274 & Protein phosphatase 1 , regulatory inhibitor subunit $14 \mathrm{~A}$ & down & 54 \\
\hline HSPA2 & 3,306 & Heat shock $70 \mathrm{kDa}$ protein 2 & down & 47 \\
\hline SLC12A2 & 6,558 & Solute carrier family 12 (sodium/potassium/chloride transporter), member 2 & up & 45 \\
\hline CRIP1 & 1,396 & Cysteine-rich protein 1 (intestinal) & down & 39 \\
\hline
\end{tabular}

${ }^{a}$ DE_State, differential expression state in colorectal cancer tissue. Up, upregulated; down, downregulated, Degree, the number of edges for a differentially expressed genes in the co-expression network.

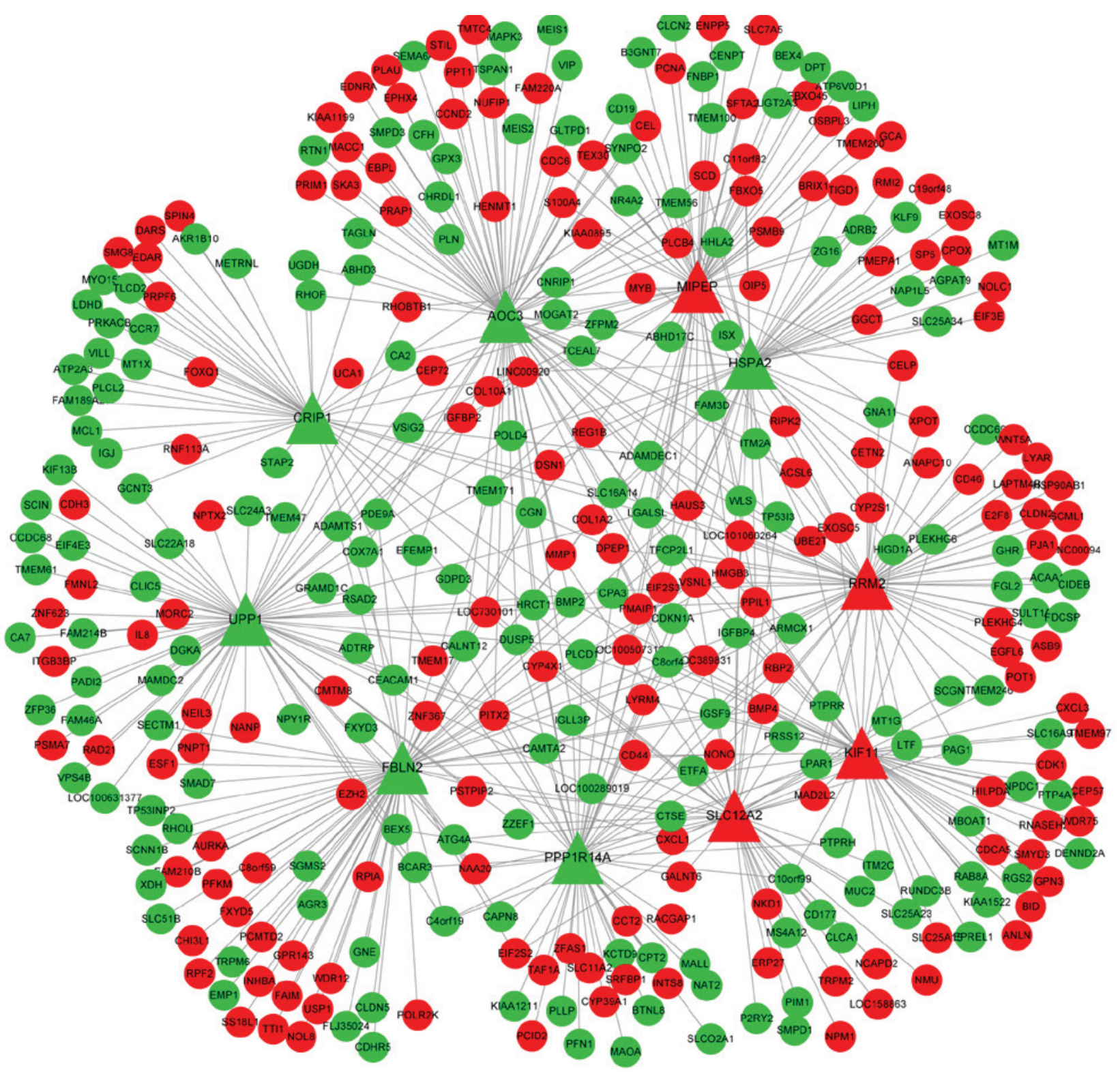

Figure 1. Co-expression network of reverse-overlapped differentially expressed genes in colorectal cancer. Red nodes represent the upregulated genes and green nodes represent the downregulated genes. Triangle nodes represent reverse-overlapped genes; circle nodes represent the differentially expressed genes. 


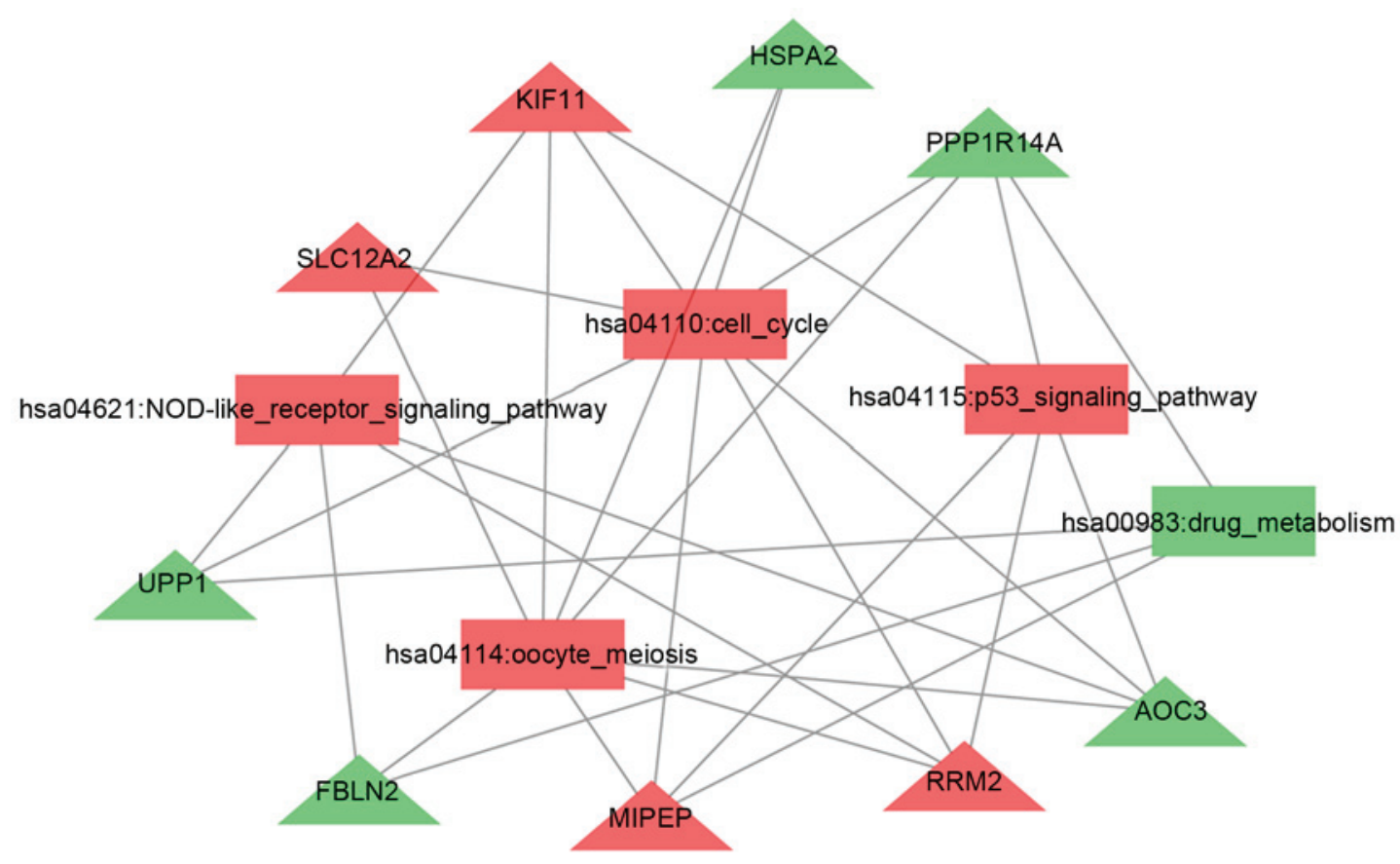

Figure 2. Sub-network of associated Kyoto Encyclopedia of Genes and Genomes signaling pathways in colorectal cancer. Red triangle nodes represent upregulated genes and green triangle nodes represent downregulated genes. Red rectangles represent upregulated gene-enriched pathways and green rectangles represent downregulated gene-enriched pathways. AOC3, amine oxidase, copper containing 3; FBLN2, fibulin 2; KIF11, kinesin family member 11; HSPA2, heat shock $70 \mathrm{kDa}$ protein 2; MIPEP, mitochondrial intermediate peptidase; PPP1R14A, protein phosphatase 1, regulatory inhibitor subunit 14A; RRM2, ribonucleotide reductase M2; SLC12A2, solute carrier family 12 (sodium/potassium/chloride transporter), member 2; UPP1, uridine phosphorylase 1.

nucleotide-binding oligomerization domain-like receptors (NLR) signaling pathways (Fig. 2).

\section{Discussion}

Significant progress has been achieved in the diagnosis and treatment of CRC. However, CRC remains the third most common cancer worldwide (16). In the present study, the mRNA expression profile of GSE32323 was downloaded and DEGs were analyzed. The DEGs in 5 CRC cell lines prior and subsequent to 5-aza-dC treatment were combined with the CRC-specific gene expression profiling array. A total of 6 reverse-overlapped DEGs were obtained. These reverse-overlapped DEGs were downregulated in the CRC tissue samples but upregulated in CRC cell lines following 5-aza-dC treatment. The CLR algorithm was employed to construct a co-expression network of CRC-specific gene expression profile and the sub-network that correlated with reverse-overlapped DEGs was selected. Furthermore, functional pathway analysis identified the reverse-overlapped DEGs enriched in a number of critical cellular pathways, including p53, cell cycle and the NLR signaling pathway.

The 6 reverse-overlapped DEGs identified in the present study were involved in a variety of cellular functions. Among them, two genes (FBLN2 and PPP1R14A) have been previously reported to be hypermethylated $(17,18)$. FBLN2, an extracellular matrix (ECM) protein, is recognized as a multifunctional binding protein (19). Due to its ability to mediate interactions between diverse ECM components, FBLN2 plays an important role in the maintenance of extracellular structures such as the basement membranes, as well as contacts between cells and $\operatorname{ECM}(20,21)$. FBLN2 has also been reported to have the opposite effects in pathological conditions including cancer. The pro-tumor effects of FBLN2 were demonstrated in pancreatic cancer cells (22); however, an increasing number of studies indicate that FBLN2 may act as an anti-angiogenic factor in various types of cancer, including nasopharyngeal carcinoma $(18,23,24)$, as well as an anti-tumor factor in breast cancer cells (25). In addition, FBLN2 has been previously demonstrated to be epigenetically silenced in B cell acute lymphoblastic leukemia (26) and methylated in breast and other epithelial cancer types (27). In the present study, FBLN2 was downregulated in the CRC tissue sample but upregulated in CRC cell lines following treatment with 5-aza-dC.

PPP1R14A was also identified to be methylated in CRC. Following treatment with 5 -aza-dC, the expression of PPP1R14A increased significantly. PPP1R14A is a phosphorylation-dependent inhibitory protein of smooth muscle myosin phosphatase activity (28), which has been reported to be an epigenetic biomarker in CRC (29). The PPP1R14A gene has also been reported to be associated with growth arrest and DNA damage (30). Following treatment with anti-cancer drugs, including Fluorouracil, PPP1R14A is upregulated (31). In addition, a previous study has demonstrated that PPP1R14A is aberrantly methylated in human esophageal squamous cell carcinoma (18) and various types of B-cell non-Hodgkin lymphoma (32).

A co-expression network based on the data of the CRC-specific gene expression profile was constructed using the CLR algorithm and the sub-network corresponding to reverse-overlapped DEGs was selected. Bias from uneven conditions of sampling, upstream regulation and inter-laboratory variations in microarray can make it difficult to infer network between genes (14). CLR algorithm increases the contrast 
between the physical interactions and the indirect associations by taking the context of each interaction and association into consideration, thus minimizing the bias from these factors (14). Therefore, the CLR algorithm is an attractive method to use for the identification of indirect links and for uncovering associations between genes within co-regulated communities. The CLR algorithm estimates a likelihood of the MI score for a particular pair of genes by comparing the MI values for that particular pair of genes to a background distribution of MI values. The most probable interactions are those whose MI scores are significantly above the background distribution of MI scores (11). Following KEGG pathway enrichment, the reverse-overlapped DEGs identified in the present study were demonstrated to be enriched in several pathways, including p53, cell cycle and NLR signaling pathways. As previously reported, p53, cell cycle and NLR receptor signaling pathways are closely associated with tumorigenesis and metastasis (33-35). The results of co-expression network analysis indicated the identified reverse-overlapped DEGs may be important tumor suppressors and are inactivated by methylation in CRC.

Compared to the previous study published by Khamas et al (9), the criteria used for selecting DEGs in the present study were different. Khamas et al (9) selected probe sets from cell lines using a combination of two criteria: Upregulation of gene expression in $\geq 4$ CRC cell lines and FC $>1.6$ in at least one cell line. In the present study, DEGs were selected if they were co-upregulated or co-downregulated in $\geq 3$ cell lines and at the same time FC> 2 in at least one cell line. Due to the difference in threshold selection for DEGs, the identified genes in the present study were different from the previously published report (9). Furthermore, a co-expression network was constructed using the CLR algorithm and the sub-network correlated with the identified genes was selected. Pathway enrichment analysis was performed to reveal the function of identified genes.

There were a number of limitations in the present study. The expression of the identified targets (FBLN2 and PPP1R14A), as well as the association of the methylation status of these genes with the development of $\mathrm{CRC}$, remains to be confirmed by future investigations.

In the present study, two silenced genes FBLN2 and PPP1R14A with abnormal methylation in CRC were identified. Furthermore, the co-expression network of identified DEGs in the CRC tissue samples was constructed by employing the CLR algorithm and a sub-network of reverse-overlapped DEGs was selected. Functional pathway analysis indicated that the identified reverse-overlapped DEGs were enriched in a number of pathways, including p53, cell cycle and NLR signaling pathway. The results of the present study may provide novel targets for the treatment of CRC.

\section{References}

1. Siegel R, Naishadham D and Jemal A: Cancer statistics, 2013. CA Cancer J Clin 63: 11-30, 2013.

2. Jemal A, Ward E and Thun M: Declining death rates reflect progress against cancer. PLoS One 5: e9584, 2010.

3. Bender CM, Pao MM and Jones PA: Inhibition of DNA methylation by 5-aza-2'-deoxycytidine suppresses the growth of human tumor cell lines. Cancer Res 58: 95-101, 1998.

4. Kim MS, Lee J and Sidransky D: DNA methylation markers in colorectal cancer. Cancer Metastasis Rev 29: 181-206, 2010.
5. Luo Y, Wong CJ, Kaz AM, Dzieciatkowski S, Carter KT, Morris SM, Wang J, Willis JE, Makar KW, Ulrich CM, et al: Differences in DNA methylation signatures reveal multiple pathways of progression from adenoma to colorectal cancer. Gastroenterology 147: 418-429.e8, 2014.

6. Zitt M, Zitt M and Müller HM: DNA methylation in colorectal cancer-impact on screening and therapy monitoring modalities? Dis Markers 23: 51-71, 2007.

7. Takai $\mathrm{N}$ and Narahara $\mathrm{H}$ : Array-based approaches for the identification of epigenetic silenced tumor suppressor genes. Curr Genomics 9: 22-24, 2008.

8. Carmona FJ and Esteller M: Epigenomics of human colon cancer. Mutat Res 693: 53-60, 2010.

9. Khamas A, Ishikawa T, Shimokawa K, Mogushi K, Iida S, Ishiguro M, Mizushima $H$, Tanaka $H$, Uetake $H$ and Sugihara K: Screening for epigenetically masked genes in colorectal cancer using 5-Aza-2'-deoxycytidine, microarray and gene expression profile. Cancer Genomics Proteomics 9: 67-75, 2012.

10. Gautier L, Cope L, Bolstad BM and Irizarry RA: affy - analysis of Affymetrix GeneChip data at the probe level. Bioinformatics 20: 307-315, 2004.

11. Smyth GK: Limma: Linear models for microarray data. In: Bioinformatics and computational biology solutions using $\mathrm{R}$ and Bioconductor. Springer, New York, NY, pp397-420, 2005.

12. Meyer PE, Lafitte F and Bontempi G: Minet: AR/Bioconductor package for inferring large transcriptional networks using mutual information. BMC Bioinformatics 9: 461, 2008.

13. Shannon P, Markiel A, Ozier O, Baliga NS, Wang JT, Ramage D, Amin N, Schwikowski B and Ideker T: Cytoscape: A software environment for integrated models of biomolecular interaction networks. Genome Res 13: 2498-2504, 2003.

14. Treviño S III, Sun Y, Cooper TF and Bassler KE: Robust detection of hierarchical communities from Escherichia coli gene expression data. PLoS Comput Biol 8: e1002391, 2012.

15. Huang da W, Sherman BT and Lempicki RA: Systematic and integrative analysis of large gene lists using DAVID bioinformatics resources. Nat Protoc 4: 44-57, 2009.

16. Benson AB III: Epidemiology, disease progression, and economic burden of colorectal cancer. J Manag Care Pharm 13 (6 Suppl C): S5-S18, 2007.

17. Hill VK: Identification of DNA Methylation Changes in Sporadic Breast and Other Cancers. University of Birmingham, 2011.

18. Jung N, Won JK, Kim BH, Suh KS, Jang JJ and Kang GH: Pharmacological unmasking microarray approach-based discovery of novel DNA methylation markers for hepatocellular carcinoma. J Korean Med Sci 27: 594-604, 2012.

19. Olin AI, Mörgelin M, Sasaki T, Timpl R, Heinegård D and Aspberg A: The proteoglycans aggrecan and Versican form networks with fibulin-2 through their lectin domain binding. J Biol Chem 276: 1253-1261, 2001.

20. Argraves WS, Greene LM, Cooley MA and Gallagher WM: Fibulins: Physiological and disease perspectives. EMBO Rep 4: 1127-1131, 2003.

21. de Vega S, Iwamoto T and Yamada Y: Fibulins: Multiple roles in matrix structures and tissue functions. Cell Mol Life Sci 66: 1890-1902, 2009.

22. Senapati S, Gnanapragassam VS, Moniaux N, Momi N and Batra SK: Role of MUC4-NIDO domain in the MUC4-mediated metastasis of pancreatic cancer cells. Oncogene 31: 3346-3356, 2012.

23. Law EW, Cheung AK, Kashuba VI, Pavlova TV, Zabarovsky ER, Lung HL, Cheng Y, Chua D, Lai-Wan Kwong D, Tsao SW, et al: Anti-angiogenic and tumor-suppressive roles of candidate tumor-suppressor gene, Fibulin-2, in nasopharyngeal carcinoma. Oncogene 31: 728-738, 2012.

24. Shuen WH and Lung ML: Fibulin-2 suppresses tumor growth and angiogenesis through the inhibition of Erk1/2 and p65 pathways in nasopharyngeal carcinoma. In: Proceedings of the AACR 104th Annual Meeting 2013. AACR, Washington, DC, p4309, 2013.

25. Fontanil T, Rúa S, Llamazares M, Moncada-Pazos A, Quirós PM, García-Suárez O, Vega JA, Sasaki T, Mohamedi Y, Esteban MM, et al: Interaction between the ADAMTS-12 metalloprotease and fibulin-2 induces tumor-suppressive effects in breast cancer cells. Oncotarget 5: 1253-1264, 2014.

26. Dunwell TL, Hesson LB, Pavlova T, Zabarovska V, Kashuba V, Catchpoole D, Chiaramonte R, Brini AT, Griffiths M, Maher ER, et al: Epigenetic analysis of childhood acute lymphoblastic leukemia. Epigenetics 4: 185-193, 2009. 
27. Hill VK, Hesson LB, Dansranjavin T, Dallol A, Bieche I, Vacher S, Tommasi S, Dobbins T, Gentle D, Euhus D, et al: Identification of 5 novel genes methylated in breast and other epithelial cancers. Mol Cancer 9: 51, 2010.

28. Eto M, Ohmori T, Suzuki M, Furuya K and Morita F: A novel protein phosphatase-1 inhibitory protein potentiated by protein kinase C. Isolation from porcine aorta media and characterization. J Biochem 118: 1104-1107, 1995.

29. Ali D, Honne H, Danielsen S, Cekaite L, Meling G, Rognum T, Lothe R and Lind G: 694 Identification of novel epigenetic biomarkers in colorectal cancer, GLDC and PPP1R14A. Eur J Cancer Suppl 8: 175, 2010.

30. Hollander MC, Zhan Q, Bae I and Fornace AJ Jr: Mammalian GADD34, an apoptosis- and DNA damage-inducible gene. J Biol Chem 272: 13731-13737, 1997.
31. Park JS, Young Yoon S, Kim JM, Yeom YI, Kim YS and Kim NS: Identification of novel genes associated with the response to 5-FU treatment in gastric cancer cell lines using a cDNA microarray. Cancer Lett 214: 19-33, 2004.

32. Bethge N, Honne H, Hilden V, Trøen G, Eknæs M, Liestøl K, Holte H, Delabie J, Smeland EB and Lind GE: Identification of highly methylated genes across various types of B-cell non-hodgkin lymphoma. PLoS One 8: e79602, 2013.

33. Sherr CJ and McCormick F: The RB and p53 pathways in cancer. Cancer cell 2: 103-112, 2002.

34. Hartwell LH and Kastan MB: Cell cycle control and cancer. Science 266: 1821-1828, 1994.

35. Lin WW and Karin M: A cytokine-mediated link between innate immunity, inflammation, and cancer. J Clin Invest 117: 1175-1183, 2007. 